\title{
Vapor deposition coating of fused silica tubes with amorphous selenium
}

N.M. Chiera ${ }^{*}, 1,2$, R. Eichler ${ }^{1,2}$, A. Vögele ${ }^{1}$, A. Türler ${ }^{1,2}$.

${ }^{1}$ University of Bern, 3012 Bern, Switzerland.

${ }^{2}$ Paul Scherrer Institute, 5232 Villigen, Switzerland.

*Corresponding author.

Postal address: Paul Scherrer Institute (PSI),

Nadine Mariel Chiera, OFLB/105

5232 Villigen PSI Switzerland

E-mail address: nadine-mariel.chiera@psi.ch.

Tel.: +41563102469

Abstract

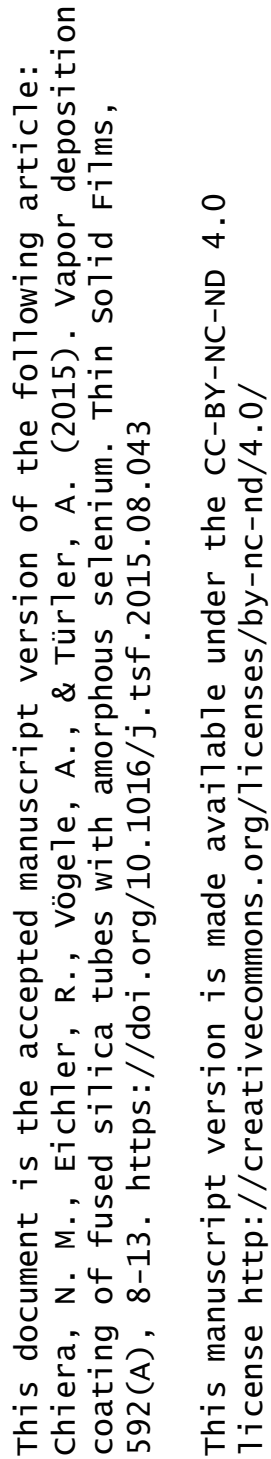

A set of optimized deposition conditions for the inner walls coating of fused silica tubes with amorphous selenium was elaborated. The method is based on the vapor transport deposition of pure elemental selenium on a cooled substrate held at liquid nitrogen temperatures. Morphological and structural examination of the deposited layer was performed by optical microscopy and X-ray diffraction studies. Neutron activated selenium was used to 
monitor the deposition pattern and its stability under high gas flows. Monte Carlo simulations allowed the estimation of the different Se species composing the amorphous phase, at the given experimental deposition conditions. The versatility of the coating method presented in this work allows for the coating of tubes of different lengths and diameters, opening the way for several applications of amorphous selenium films in various fields.

\section{Keywords}

coating; column; diffusion; amorphous selenium; selenium; thin layer; tube; vapor transport deposition.

\section{Abbreviations}

In the present work amorphous selenium is referred to as "a-Se".

\section{Introduction}

At room temperature and atmospheric pressure, the major allotropic forms in which selenium exists are crystalline (trigonal, $\alpha-, \beta-, \gamma$-monoclinic), amorphous (red, brown and black Se), and vitreous selenium [1]. The most thermodynamically stable form is trigonal selenium, also known as grey, hexagonal, or metallic selenium. Trigonal Se consists of polymeric Sen helical chains, in which contiguous atoms form single covalent bonds [2], and it is less chemically active than the other allotropes [3]. The structure of the amorphous phase has been long discussed and various models have been proposed [4- 8]. It is generally accepted that the amorphous allotrope consists of a mixture of selenium ring-like molecules of different size 
and branched chains of diverse length, interconnected to each other by Van der Waals forces $[9,10]$. At slow heating of the red amorphous selenium at temperatures higher than $37^{\circ} \mathrm{C}$, this allotrope undergoes a phase change, transforming gradually to the black amorphous one [11, 12].

During the last decades, amorphous selenium (a-Se) acquired high importance in the field of technology $[13,14,15,16]$, in the biomedical area $[17,18]$, as well in the environmental and safety sectors [19, 20]. For the production of a-Se as thin films or nanopowders different techniques were applied, such as chemical vapor deposition [21], chemical bath deposition [22], vacuum evaporation [23], etc. However, all of these techniques were conducted on flat substrates, limiting the uses of this chalcogen.

In the present work, a vapor transport and fast quenching deposition method is used for the coating of inner walls of fused silica tubes with amorphous selenium - in particular, with the red amorphous allotrope - since the quenching of selenium vapors at low temperatures leads to the production of amorphous (red) selenium [12, 24, 25].

The here presented technique does not require sophisticate instrumentation; unlike solution growth techniques, electrodeposition or chemical bath deposition, this system can be easily kept free from impurities like water, organic substances and oxygen in order to avoid their incorporation into the deposited selenium layers, which typically influences their physical and chemical properties [26, 27]. Using this method, a-Se coatings of the desired thicknesses can be prepared, just by varying the selenium amount used as starting material. Furthermore, the proposed method is safe, since it avoids the use or production of poisonous compounds like $\mathrm{H}_{2} \mathrm{Se}$ and $\mathrm{SeO}_{2}$, it is reproducible and inexpensive.

Since a-Se is a metastable state which undergoes a phase change to the most thermodynamically stable trigonal selenium, a set optimized vapor transport deposition conditions was elaborated in order to limit the spontaneous a-Se crystallization process. 
Recently, the coating technique discussed here was applied for the coverage of the inner walls of quartz columns with amorphous selenium for radiochemical studies [28].

\section{Experimental details}

\subsection{Pretreatment of the fused silica tubes}

Fused silica tubes (i. $\varnothing=4 \mathrm{~mm}$; length $=1 \mathrm{~m}$ ) were rinsed with $\mathrm{MeOH}$ (anhydrous, $\geq 99.8 \%$, Sigma-Aldrich), AcOH (VWR) and EtOEt (anhydrous, $\geq 99.0 \%$, Sigma-Aldrich) in the mentioned order, and dried under a pure N2 gas flow.

\subsection{Selenium deposition}

A fused silica tube (3) is placed in the vapor transport system as shown in Fig. 1. A high purity stream of He carrier gas is passed through a tantalum getter-oven (2) kept at $1273 \mathrm{~K}$ in order to remove $\mathrm{H}_{2} \mathrm{O}$ and $\mathrm{O}_{2}$ traces. The content of $\left(\mathrm{H}_{2} \mathrm{O}+\mathrm{O}_{2}\right)$ in the gas, measured with a trace oxygen sensor (Ionic Systems TOS 3.0), is $0.5 \mathrm{ppm}$ after 2 hours of running the carrier gas. The He gas flow rate of $300 \mathrm{ml} / \mathrm{min}$, established during the experiments, is controlled by a mass flow controller (1) (Series 5850E, Brooks Instruments) and measured with a Buck calibrator (A.P. Buck, Inc.). High purity (>99.999\%) elemental grey Se (Fluka Chemicals) is volatilize inside the fused silica column in the sublimation oven (4) kept at 653 $\mathrm{K}$ ( internal temperature: $620 \mathrm{~K}$ ), and transported by the carrier gas to the liquid nitrogen cooled fused silica column, where the deposition of the a-Se takes place. A third oven (5), kept at $653 \mathrm{~K}$ (column internal temperature: $620 \mathrm{~K}$ ), is placed adjacent to the liquid nitrogen cooling tank (6), and is used as a thermal buffer. The temperature gradient established along 
the fused silica tube is shown in Fig. 2. All the resistive ovens are connected to VARIAC autotransformers type W20HMT3, and the temperatures are measured with a Voltcraft K202 digital thermometer connected to K-type thermocouples placed inside the tubular ovens. After the total selenium evaporation, ovens (4) and (5) are switched off and the system is allowed to reach room temperature under the dry He gas flow. The non-deposited selenium vapor is captured in a charcoal trap placed at the end of the fused silica tube (7).

\subsection{Characterization}

\subsubsection{Optical microscopy}

The morphology and the homogeneity of the deposited a-Se were examined by optical microscopy using a Bresser microscope (magnification: 4-100x). Photomicrographs were taken using a DinoEye ${ }^{\circledR}$ digital ocular camera.

\subsection{2. ${ }^{75}$ Se deposition}

In order to characterize quantitatively the deposition pattern of the amorphous selenium and its stability on the substrates under high gas flows, radioactive ${ }^{75}$ Se containing samples $(\mathrm{t} 1 / 2=119.78$ days; main $\gamma$-lines $=136 \mathrm{keV}(59.2 \%), 265 \mathrm{keV}(59.8 \%))$ were used as tracer. ${ }^{75}$ Se samples were produced by irradiation of the initial elemental grey Se (purity > 99.999\%, Fluka Chemicals) with thermal neutrons at the neutron activation rabbit system installed at the spallation neutron source SINQ at the Paul Scherrer Institute. The method of deposition was the same as for the non-irradiated selenium. The deposition of the radioactive 
${ }^{75}$ Se isotope along the fused silica column was determined via measuring the specific $\gamma$ activity with a high-purity germanium $\gamma$-ray detector collimated by a lead shield with a 1 $\mathrm{cm} \times 0.5 \mathrm{~cm}$ window. Thus, the data acquisition and analysis system Canberra Genie $2 \mathrm{k}^{\circledR}$ in conjunction with standard spectroscopy electronics enabled the quantification of the ${ }^{75} \mathrm{Se}$ activity in each centimeter of the column.

\subsubsection{X-ray diffraction}

Selected sections of the fused silica tube coated with selenium were weighed and ground to a fine powder using an agate mortar. The obtained powder samples were put into black plastic microtubes, and stored at 296 K. X-ray diffraction (XRD) studies were carried out with a Bruker AXS D8 Advance X-Ray Diffractometer using $\mathrm{Cu}-\mathrm{K} \alpha$ radiation $(\mathrm{E}=8047.8 \mathrm{eV})$ in the $2 \theta$ range from $5^{\circ}$ to $90^{\circ}$.

\section{Results and discussion}

\subsection{Morphology of the deposited Se}

At the beginning of the gradient of temperature $(\mathrm{T}=483 \mathrm{~K})$, between the buffer oven and the liquid nitrogen cooling (i.e. $\mathrm{cm} 22$ in Fig. 2), characteristic trigonal selenium needleshape crystals are formed (Fig. 3.a). The XRD study confirms the trigonal structure (Fig. 3.b). At $\mathrm{T} \leq 363 \mathrm{~K}$ (i.e. after $\mathrm{cm} 23$ in Fig. 2), in perfect agreement with the ceiling temperature of $\mathrm{T}=356 \mathrm{~K}$ [29], the structure of the deposited selenium is non-crystalline. In fact, the fast quenching rate and the relatively low temperatures do not allow for a polymerization and 
a subsequent crystallization, yielding predominantly the amorphous phase. Thus, from $363 \mathrm{~K}$ to 203 K (i.e. cm 23-26 in Fig. 2) a thick amorphous selenium film (Fig. 4.a) is deposited. The XRD analysis shows its amorphous structure (Fig. 4.b). Below 203 K (i.e. after cm 26 in Fig. 2) the deposition of a thin a-Se layer occurs (Fig. 5.a). The absence of crystal diffraction peaks in the XRD plot confirms the amorphous structure (Fig. 5.b).

\subsection{Parameters influencing the Se deposition}

\subsubsection{Evaporation source temperature}

In the vapor phase, selenium exists as a mixture of $\operatorname{Sei}(\mathrm{g})$ species with $\mathrm{i}=1$ to 12 , the ratio of which depends on the vapor source temperature. Until $\mathrm{T}=620 \mathrm{~K}$ the main components in the vapor phase are Se5 and Se6 species [30]. The high stability of these molecules [31, 32], leads to the deposition of a stable a-Se layer, which will manifest a slow crystallization rate (i.e. the phase change to the amorphous allotrope to the trigonal one) at room temperature, if kept in a dark, inert environment (e.g. under an Ar atmosphere), as shown in previous crystallization studies [33].

\subsubsection{Gas carrier}

An important factor in the amorphous selenium deposition layer formation is the diffusion of the species in the gas phase. Among the most common inert gas carriers $\left(\mathrm{He}, \mathrm{Ar}, \mathrm{N}_{2}\right)$, helium was chosen due its low density and molecular weight. The use of He as carrier gas leads to a faster diffusion of the small molecules like $\mathrm{Se}_{2}, \mathrm{Se}_{3}, \mathrm{Se}_{4}$ which, being the most reactive ones $[31,32]$, are the major responsible for the crystallization. 


\subsection{Discussion of the Se deposition}

The elemental selenium deposition pattern follows a spontaneous vapor deposition according to the temperature dependence of the vapor pressure [25]; however, since the system is in a non- equilibrium condition, at $\mathrm{T} \leq 203 \mathrm{~K}$, where the a-Se deposition takes place (i.e. after $\mathrm{cm} 26$ in Fig. 2), the deposition pattern shows a deviation from the expected equilibrium (Fig. 6). The difference between the calculated amount of Se expected to be evaporated at $\mathrm{T}=620 \mathrm{~K}$ and the experimental amount of Se evaporated gives a $20 \%$ mass saturation of the He carrier gas. The deposition of the a-Se layer depends on a diffusioncontrolled process of the different selenium species coexisting in the vapor phase. In this specific non-equilibrium case, the Chapman-Enskog theory [34] as well the Gilliland method, which relies on the hard spheres model, cannot be applied. In order to describe the diffusion process at temperatures below $203 \mathrm{~K}$ and at the applied experimental conditions, Monte Carlo simulations [35] were performed (Fig. 7). This powerful tool allows the description of the interaction kinetics of a single volatile atomic or molecular species in the gas phase with the column surface, in terms of an adsorption- desorption equilibrium or in the case of macroscopic amounts a sublimation / desublimation equilibrium. In the current case the reactivity of the selenium species in the gas phase with the surface is thermodynamically best described by the desublimation enthalpy of each Se species involved in the deposition [36]. The simulations take into account experimental parameters like the flow rate, the type of carrier gas, the temperature and pressure applied, the column dimensions, randomizing both the time in flight of the volatile specie in the gas phase and its residence time in the adsorbed state. For the Monte-Carlo simulations, species existing at the evaporation temperature of $620 \mathrm{~K}$ [30], and a total pressure of $10^{5} \mathrm{~Pa}$ were considered. Interactions between the different 
species (e.g. polymerization reactions) are not included. The fitting of the simulated deposition to the experimental one was obtained applying a correction factor $\mathrm{A}$ to the estimated expectation value for the diffusion coefficient calculated by the empirical Gilliland equation [37]:

(1) $D=A \frac{0.0043 T^{3 / 2}\left(\frac{1}{M S e}+\frac{1}{M g}\right)^{1 / 2}}{P\left(V s e^{1 / 3}+V g^{1 / 3}\right)^{2}} \quad$ with $\mathrm{A}=6$ for $\mathrm{T}>203 \mathrm{~K}$, and $\mathrm{A}=0.3$ for $\mathrm{T} \leq 203 \mathrm{~K}$

where $\mathrm{T}$ is the temperature $(\mathrm{K}), \mathrm{M}_{\mathrm{Se}}$ and $\mathrm{M}_{\mathrm{g}}$ are the molecular weights respectively of the selenium species and the carrier gas $(\mathrm{g} / \mathrm{mol}), \mathrm{P}$ is the pressure (atm), $\mathrm{V}_{\mathrm{Se}}$ and $\mathrm{V}_{\mathrm{g}}$ are the molar volumes respectively of the selenium species and the carrier gas $\left(\mathrm{cm}^{3} / \mathrm{mol}\right)$.

The simulated composition of the deposited amorphous selenium layer shows $\mathrm{Se}_{6}$ as the major component of the a-Se layer. The difference between the Monte Carlo simulations and the experimental amount of a-Se deposited can be explained as an underestimation of the Sei species with $\mathrm{i}>8$ in the assumed composition of the selenium vapor phase, calculated from mass spectrometry studies [30]. In fact, heavy species, being slower in the diffusion process towards the quartz walls, would be better transported by the gas flow to the last part of the column, where they would deposit.

3.4. Application of the coating technique to tubes with different radii and lengths

The deposition efficiency can be expressed in terms of a dimensionless diffusion parameter, $\Delta[38]:$

(2) $\Delta=\frac{D L}{4 U R^{2}}$ 
where $\mathrm{D}$ is the diffusion coefficient $\left(\mathrm{cm}^{2} / \mathrm{s}\right)$ at $\mathrm{T} \leq 203 \mathrm{~K}$ of the major Se species $\left(\mathrm{Se}_{6}\right)$ composing the amorphous selenium phase, $\mathrm{L}$ is the tube length $(\mathrm{cm}), \mathrm{U}$ is the average flow velocity $(\mathrm{cm} / \mathrm{s})$, and $\mathrm{R}$ is the tube radius $(\mathrm{cm})$.

Keeping the same dimensionless parameter $\Delta$ is possible to apply the here presented coating technique to tubes with different radii and lengths.

3.5. Adhesion of the amorphous selenium coating to the quartz substrate

The fused silica tube section corresponding to the amorphous selenium deposition from $\mathrm{cm} 26$ to $\mathrm{cm} 37$ in Fig. 2 - labeled with ${ }^{75} \mathrm{Se}$ - was cut out and exposed to a $1 \mathrm{~L} / \mathrm{min}$ He gas flow for $60 \mathrm{~h}$ at room temperature. No re-volatilization or particle movement of the amorphous selenium was observed (Fig. 8).

\section{Conclusions}

The optimum experimental conditions for coating the inner walls of fused silica tubes with aSe thin films (thickness $\sim 100 \mathrm{~nm}$ ) are presented. From tracer experiments with ${ }^{75} \mathrm{Se}$ the composition of the deposited amorphous Se layer was estimated, revealing the Se 5 and Se6 species as the major components. The storage of the in this manner coated fused silica tubes in a dark and inert environment, at temperatures lower than the glass transition point ( $\mathrm{Tg} \sim 298 \mathrm{~K}$ ), allows for their utilization until the material properties change, due to the phase transformation to the thermodynamically stable trigonal selenium by ageing. Different coating thicknesses can be obtained by varying the initial amount of the elemental selenium in the 
sublimation source. This coating method can be also applied to tubes of other diameters or lengths, keeping the same diffusion parameter $\Delta$ as for the here presented tubes. Additionally, the use of ${ }^{75} \mathrm{Se}$ tracer allowed demonstrating long-term stability against revolatilization at room temperature and gas flow speeds of the order of $1.3 \mathrm{~m} / \mathrm{s}$.

References

[1] V.S. Minaev, S.P. Timoshenkov, V. V. Kalugin, Structural and phase transformations in condensed selenium, J. Optoelectron. Adv. Mater. 7 (2005) 1717-1741.

[2] H. Fujisaki, J. B. Westmore, A. W. Tickner, Mass spectrometric study of subliming selenium, Can. J. Chem. 44 (1966) 3063-3071.

[3] V. J. Molinski, G. W. Leddicotte, Radiochemistry of selenium, National Academies, New York, 1965.

[4] J. Robertson, A new model for the structure of amorphous selenium, Philos. Mag. 34 (1976) $13-31$.

[5] G. Lucovsky, Selenium, the amorphous and liquid states, in: E. Gerlach, P. Grosse (Eds.), The Physics of Selenium and Tellurium, Proceedings of the International Conference on the Physics of Selenium and Tellurium, Königstein, Fed. Rep. of Germany, Springer-Verlag, New York, 1979, pp. 178-192.

[6] A. A. Baganich, V. I. Mikla, D. G. Semak, A. P. Sokolov, A. P. Shebanin, Raman scattering in amorphous selenium molecular structure and photoinduced crystallization, Phys. Status Solidi B (1991), 297-302.

[7] R. Böhmer, C. A. Angeli, Elastic and viscoelastic properties of amorphous selenium and identification of the phase transition between ring and chain structures, Phys. Rev. B 48 (1993) 5857-5864. 
[8] J. Berashevich, A. Mishchenko, A. Reznik, Two-Step photoexcitation mechanism in amorphous Se, Phys. Rev. Appl. 1(2014) 034008.1-034008.8.

[9] L. Yu. Kupriyanov, Semiconductor sensors in physico-chemical studies, in: S. Middelhoek (Eds.), Handbook of sensors and actuators, Elsevier, Amsterdam, 1996, pp. 368-369.

[10] D. Caprion, H. R. Schober, Structure and relaxation in liquid and amorphous selenium, Phys. Rev. B 62 (2000) 3709-3716.

[11] V. S. Minaev, Polymorphous-crystalloid structure and relaxation processes in some chalcogenide glass-forming substances, J. Optoelectron. Adv. Mater. 3 (2001) 233-248.

[12] H. Gobrecht, Transformations of red amorphous and monoclinic selenium, J. Phys. Chem. Solids 31 (1970) 2148-2151

[13] C. Haugen, S. O. Kasap, J. Rowlands, Charge transport and electron-hole-pair creation energy in stabilized a-Se x-ray photoconductors, J. Phys. D: Appl. Phys. 32 (1999) 200-207. [14] M. Kastner, Bonding bands, lone-pair bands, and impurity states in chalcogenide semiconductors, Phys. Rev. Lett. 28 (1972) 355-357.

[15] S. Abbaszadeh, K. Wang, K. S. Karim, Low Dark-Current Lateral Amorphous-Selenium Metal-Semiconductor-Metal Photodetector, Electron Device Lett. 32 (2011) 1263-1265.

[16] S. Kasap, J. B. Frey, G. Belev, O. Tousignant, H. Mani, J. Greenspan, L. Laperriere, O. Bubon, A. Reznik, G. DeCrescenzo, K.S. Karim, J. A. Rowlands, Amorphous and polycrystalline photoconductors for direct conversion flat panel x-ray image sensors, Sensors, $11(2011), 5112-5157$.

[17] J. F. Ramos, T. J. Webster, Cytotoxicity of selenium nanoparticles in rat dermal fibroblasts, Int. J. Nanomedicine, 7 (2012) 3907-3914.

[18] P. A. Tran, T. J. Webster, Selenium nanoparticles inhibit Staphylococcus aureus growth, Int. J. Nanomedicine, 6 (2011) 1553-1558.

[19] N. C. Johnson, et al., Mercury vapor release from broken compact fluorescent lamps and 
in situ capture by new nanomaterial sorbents, Environ. Sci. Technol. 42 (2008) 5772-5778.

[20] N. Ralston, Nanomaterials: Nano-selenium captures mercury, Nat. Nanotechnol. 3 (2008) $527-528$.

[21] P. Nagels, E. Sleeckx, R. Callaerts, L. Tichy, Structural and optical properties of amorphous selenium prepared by plasma-enhanced CVD, Solid state Commun. 94 (1995) 4952.

[22] K. Bindu, et al., Amorphous selenium thin films prepared using chemical bath deposition: optimization of the deposition process and characterization, Semicond. Sci. Technol. 17 (2002) $270-274$

[23] D. A. Buckley, F. G. Belli, J. H. Lennon, Vacuum deposition of amorphous selenium on polymeric substrates II. Use of a temperature-gradient vacuum coating device, Surf. Technol. 9 (1979) 241-252.

[24] R. Steudel, E. Strauss, Homocyclic selenium molecules and related cations, in: G. Meurant (Eds.), Advances in inorganic chemistry and radiochemistry 28, Academic press, Orlando, 1984, p. 139.

[25] B. Wunderlich, H. Shu, The crystallization and melting of selenium, J. Cryst. Growth 48 (1980) 227-239.

[26] F. Jansen, Effects of oxygen and chlorine on the interfacial crystallization of amorphous selenium films, J. Vac. Sci. Technol. 18 (1981) 215-218.

[27] A. Legros, M. Shi, A. Mouton, A. Selmani, Effect of water impurity on the crystallization of vacuum evaporated Se, J. Appl. Phys. 78 (1995) 3048-3051.

[28] N. Chiera, R. Eichler, A. Türler, Towards selenides of the superheavy elements copernicium and flerovium, PSI Annual report (2013) 7.

[29] B. Wunderlich, Macromolecular physics Vol. 2, Elsevier, New York, 2012.

[30] Å. Olin, B. Noläng, L. Öhman, E. Osadchii, E. Rosén, Chemical thermodynamics of 
selenium, OECD Nuclear Energy Agency, Issy-les-Moulineaux, 2005.

[31] Z. Q. Li, J. Z. Yu, K. Ohno, Electronic states and stability of selenium clusters, Phys. Rev. B 52(1995) 1524-1527.

[32] J. Berkowitz, W. A. Chupka, Equilibrium composition of selenium vapor; thermodynamics of the vaporization of HgSe, CdSe, and SrSe, J. Chem. Phys. 45 (1966) 42894302.

[33] J. P. Audiere, C. Mazieres, J. C. Carballes, Ageing and crystallization of non-crystalline Se thin films: A tentative structural model, J. Noncryst. Solids 34 (1979) 37-51.

[34] S. Chapman, T. G. Cowling, The mathematical theory of non-uniform gases: An account of the kinetic theory of viscosity, thermal conduction and diffusion in gases, Cambridge University Press, Cambridge, 1970.

[35] I. Zvara, Simulation of thermochromatographic processes by the Monte Carlo method, Radiochim. Acta. 38 (1985) 95-102.

[36] R. Viswanathan, R. Balasubramanian, D. Darwin Albert Raj, M. Sai Baba, T. S. Lakshmi Narasimhan, Vaporization studies on elemental tellurium and selenium by Knudsen effusion mass spectrometry, J. Alloy Compd. 603 (2014) 75-85.

[37] N. H. Chen, D. F. Othmer, New generalized equation for gas diffusion coefficient, J. Chem. Eng. Data 7 (1962) 37-41.

[38] H. Shi, C. Kleinstreuer, Z. Zhang, C. S. Kim, Nanoparticle transport and deposition in bifurcating tubes with different inlet conditions, Phys. Fluids. 16 (2004) 2199-2213. 
List of figures and figures captions

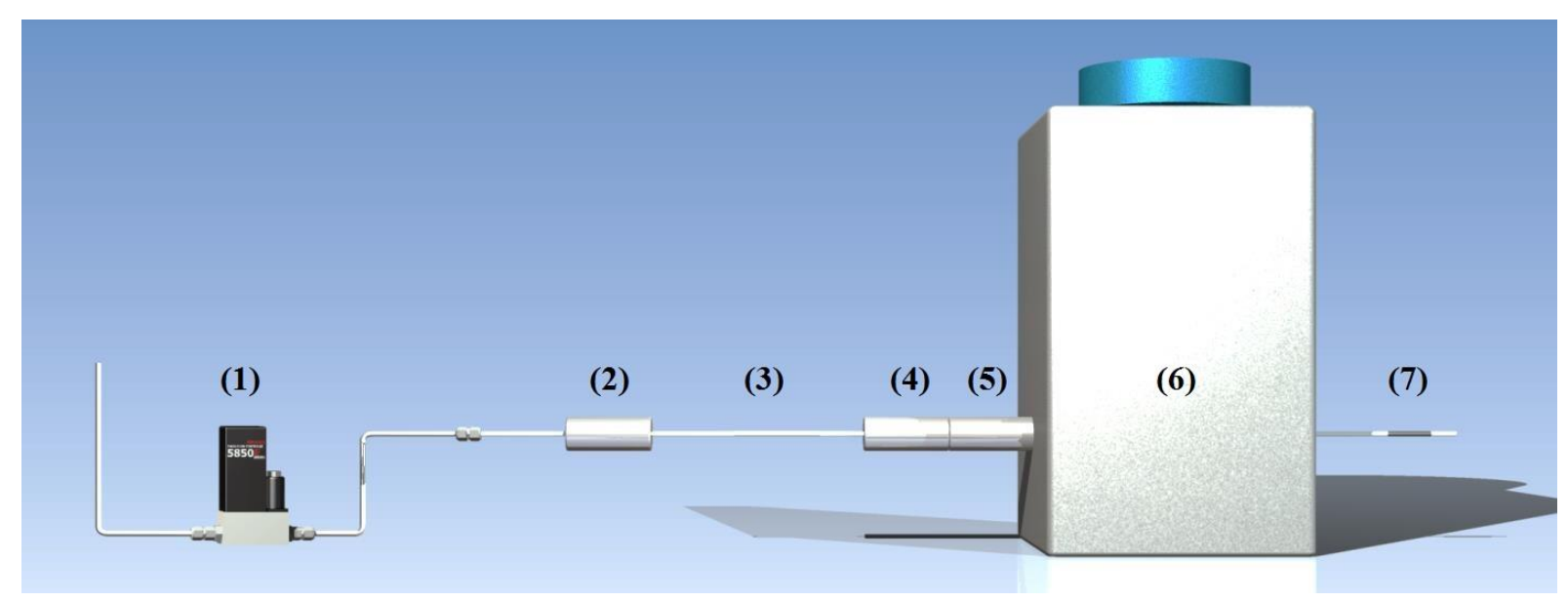

Figure 1. Sketch of the setup used for volatilization and deposition of selenium: The He gas flow regulated by a mass flow controller (1) passes through a tantalum getter oven (2) kept at $1273 \mathrm{~K}$. There are no connections between the tantalum getter oven and the fused silica tube (3) in order to minimize the oxygen and water intake. A high purity pellet of elemental 
selenium $(\sim 0.08 \mathrm{~g})$ is placed inside the fused silica tube, where the sublimation oven (4) is positioned. After 2 hours of flushing the setup by dried He gas, the oven (5) is switched on and the liquid nitrogen tank (6) is filled. After 1.5 hours the temperature gradient is established and the sublimation oven (4) can be switched on. The evaporation of $\sim 0.08 \mathrm{~g}$ of selenium takes about $8 \mathrm{~h}$. The selenium which is not deposited in the fused silica column is captured in the charcoal trap (7). 


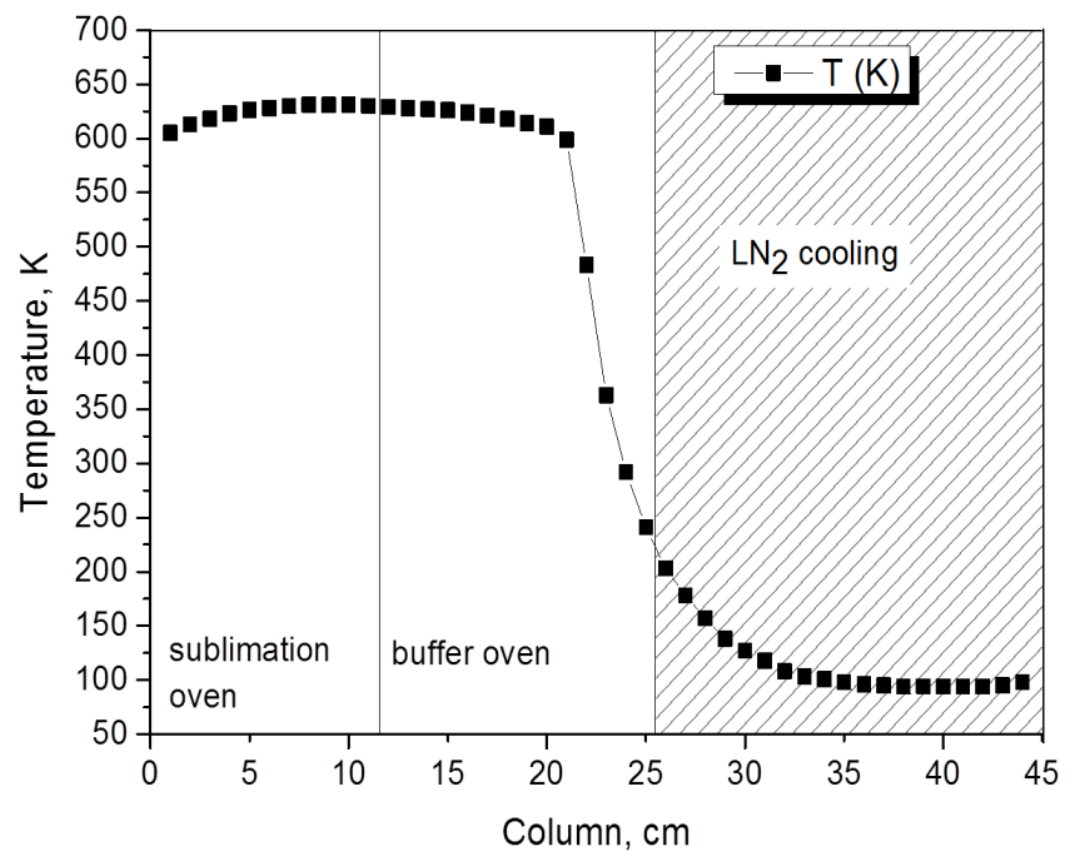

Figure 2. The temperature gradient established inside the fused silica tube. 


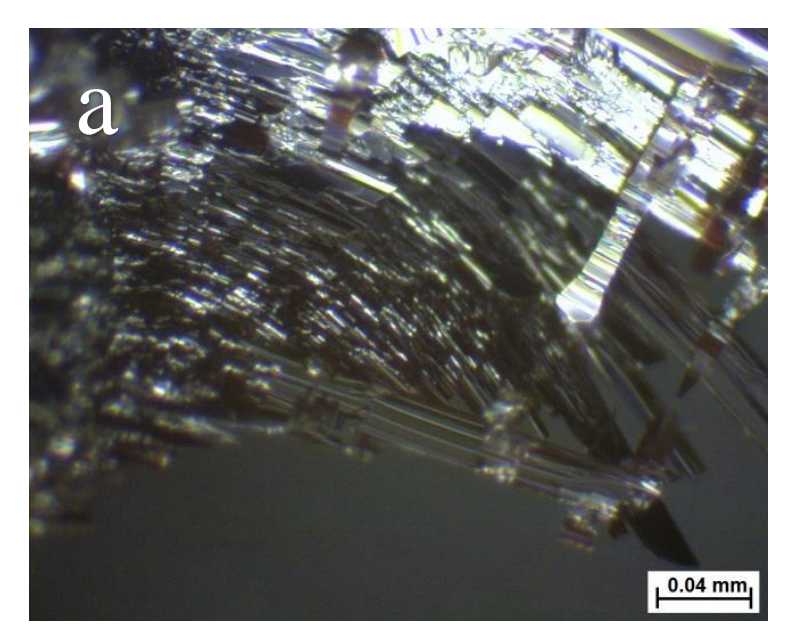




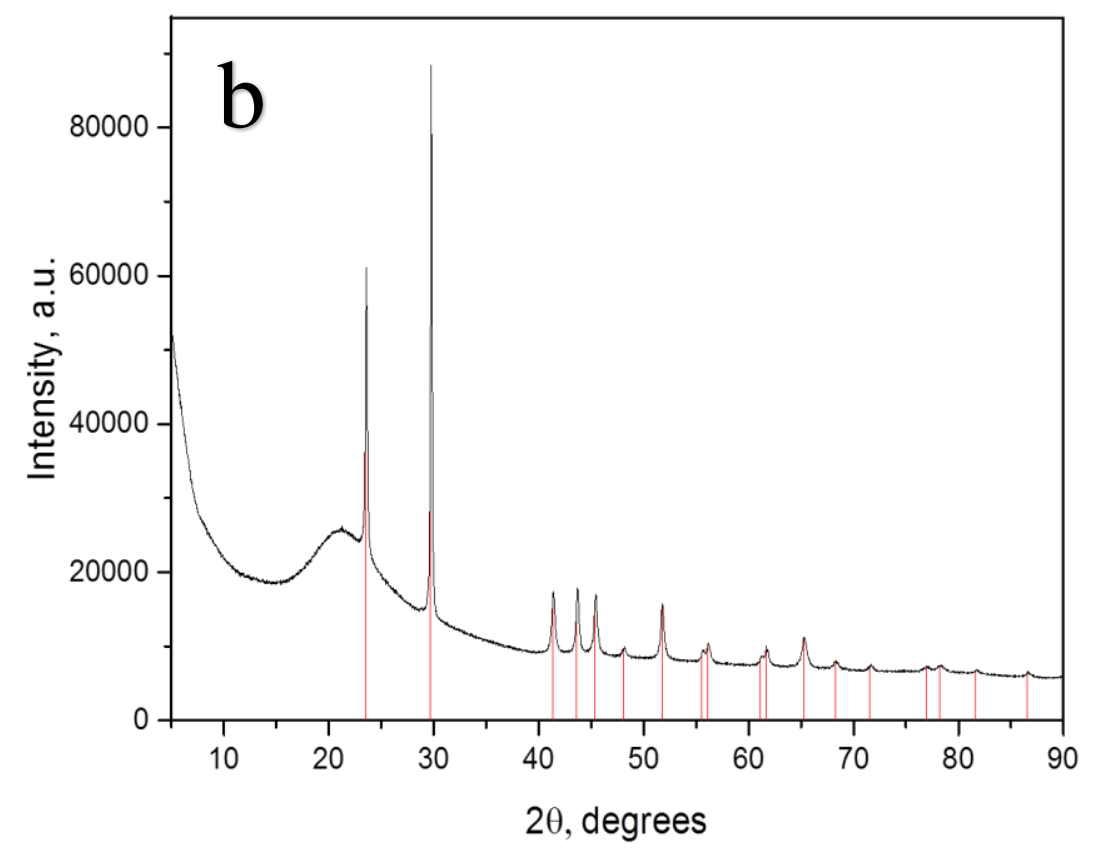

Figure 3. a) Optical microscopy image under white light-emitting diode illumination of the selenium typically deposited at $483 \mathrm{~K}$. The scale bar is $40 \mu \mathrm{m}$. b) X-ray diffraction 
spectrum of the selenium deposited at $483 \mathrm{~K}$. The broad peak from 15 to 23 degrees corresponds to the air scattering. The fitting with the Bruker X-ray diffraction database for trigonal selenium is indicated.

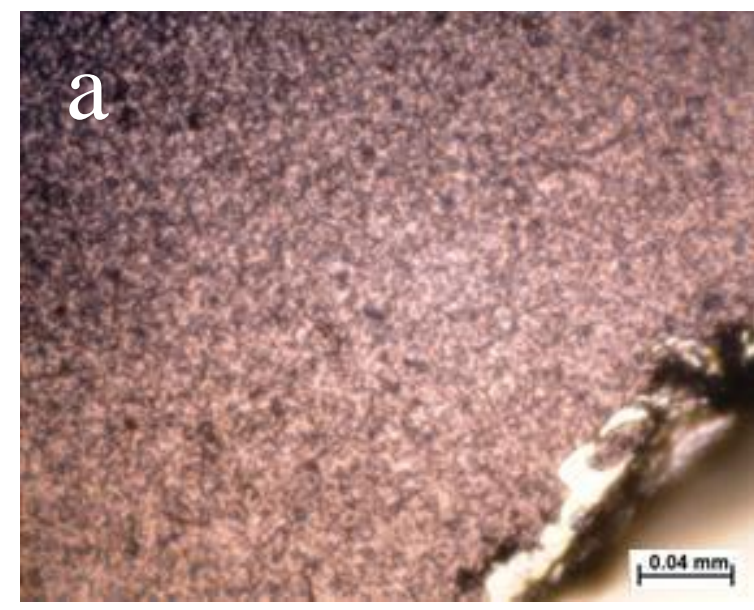




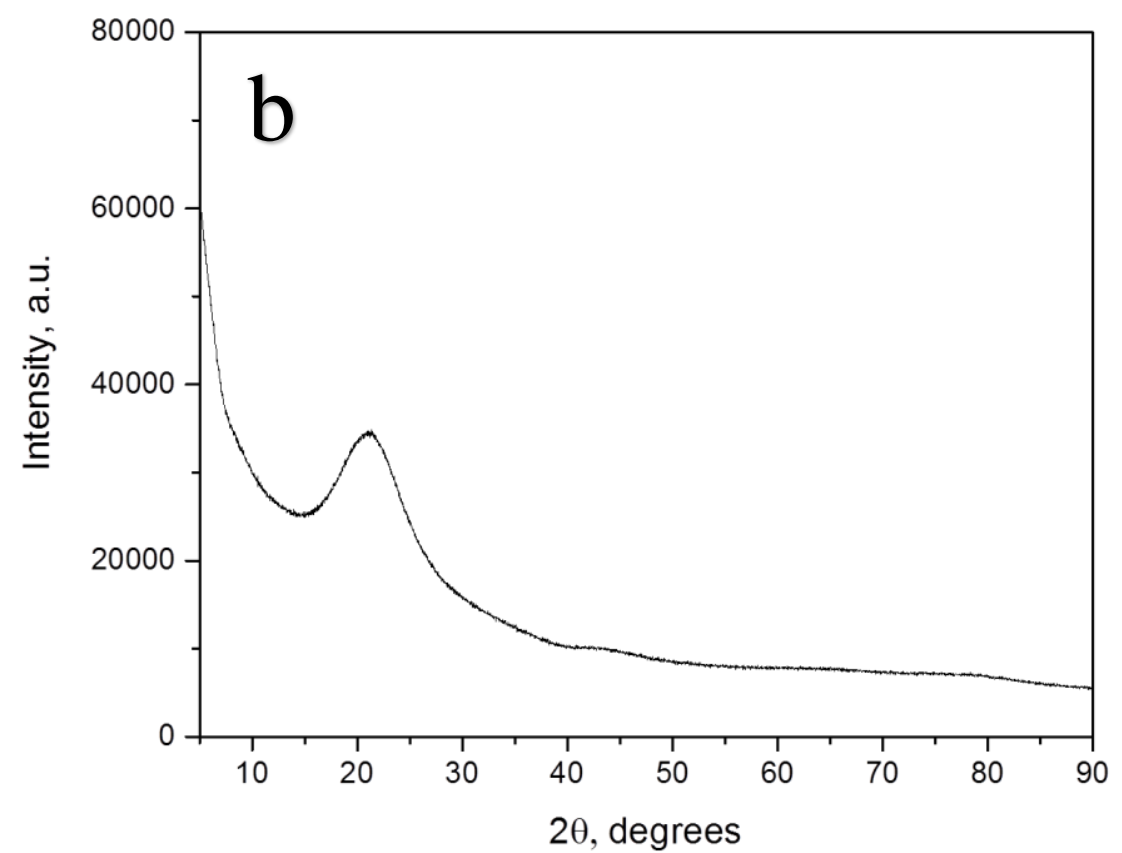

Figure 4. a) Optical microscopy image of the thick amorphous selenium deposited in the temperature range from $363 \mathrm{~K}$ to $203 \mathrm{~K}$, using a $50 \mathrm{~W}$ tungsten microscope light for 
illumination. The thickness of the deposited layer, as deduced from the ${ }^{75}$ Se pattern deposition, is $6 \mu \mathrm{m}$. Scale bar: $40 \mu \mathrm{m}$. b) X-ray diffraction pattern of the thick selenium film deposited in the temperature range from $363 \mathrm{~K}$ to $203 \mathrm{~K}$. The broad peak from 15 to 23 degrees corresponds to the air scattering.

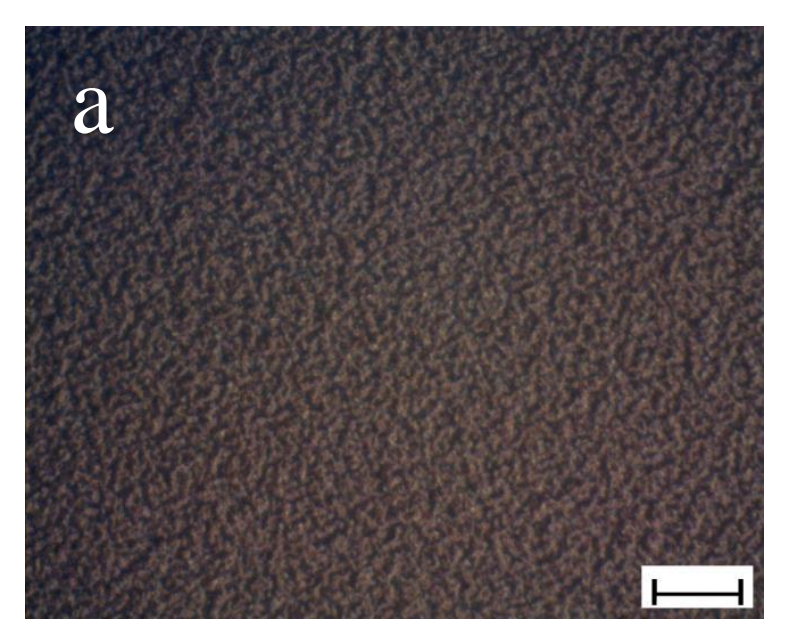




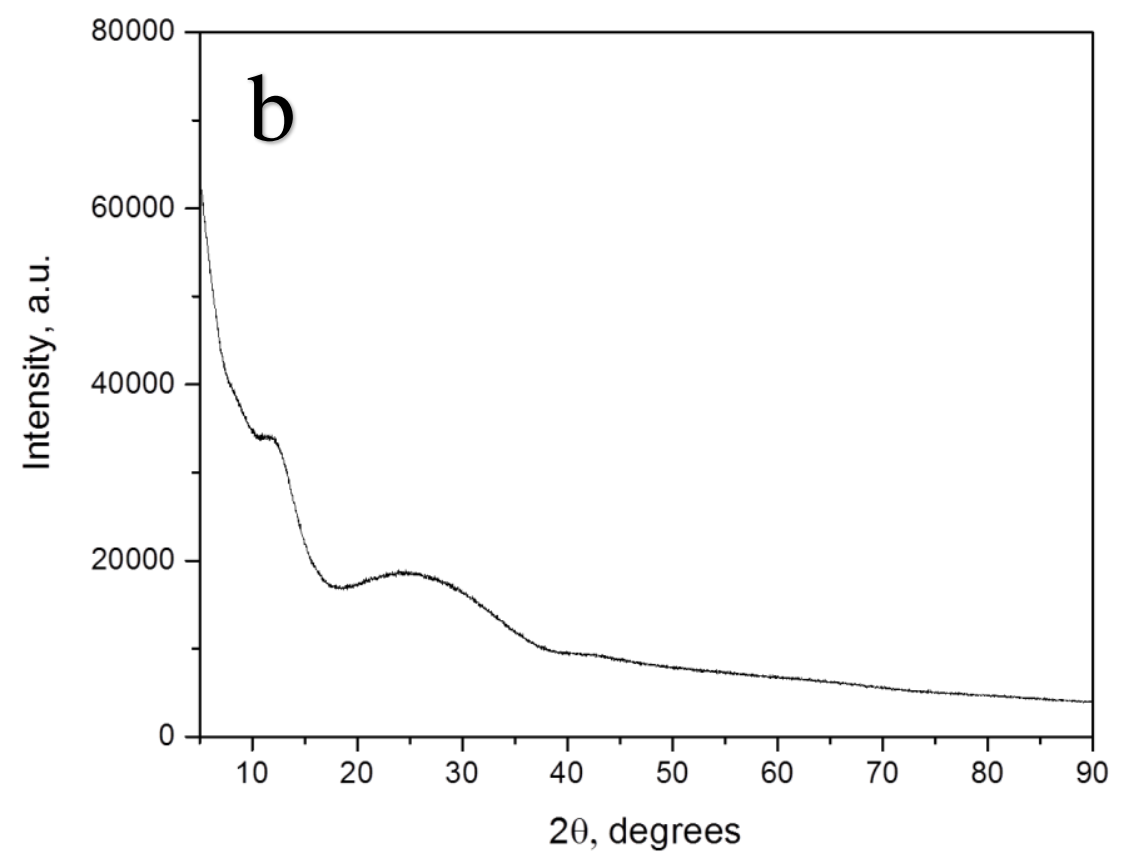

Figure 5. Optical microscopy images of the amorphous selenium deposited below 203 K. a)

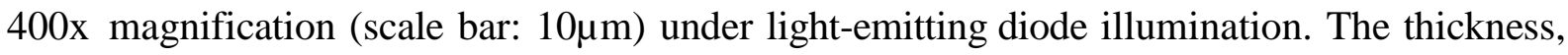


deduced from the ${ }^{75}$ Se pattern deposition, of the here presented amorphous selenium coating is $100 \mathrm{~nm}$. b) X-ray diffraction pattern of the amorphous selenium deposited below $203 \mathrm{~K}$.

The broad peak from 9 to 14 degrees corresponds to the diffraction of selenium micro and nano particles. The broad peak from 15 to 23 degrees corresponds to the air scattering. 


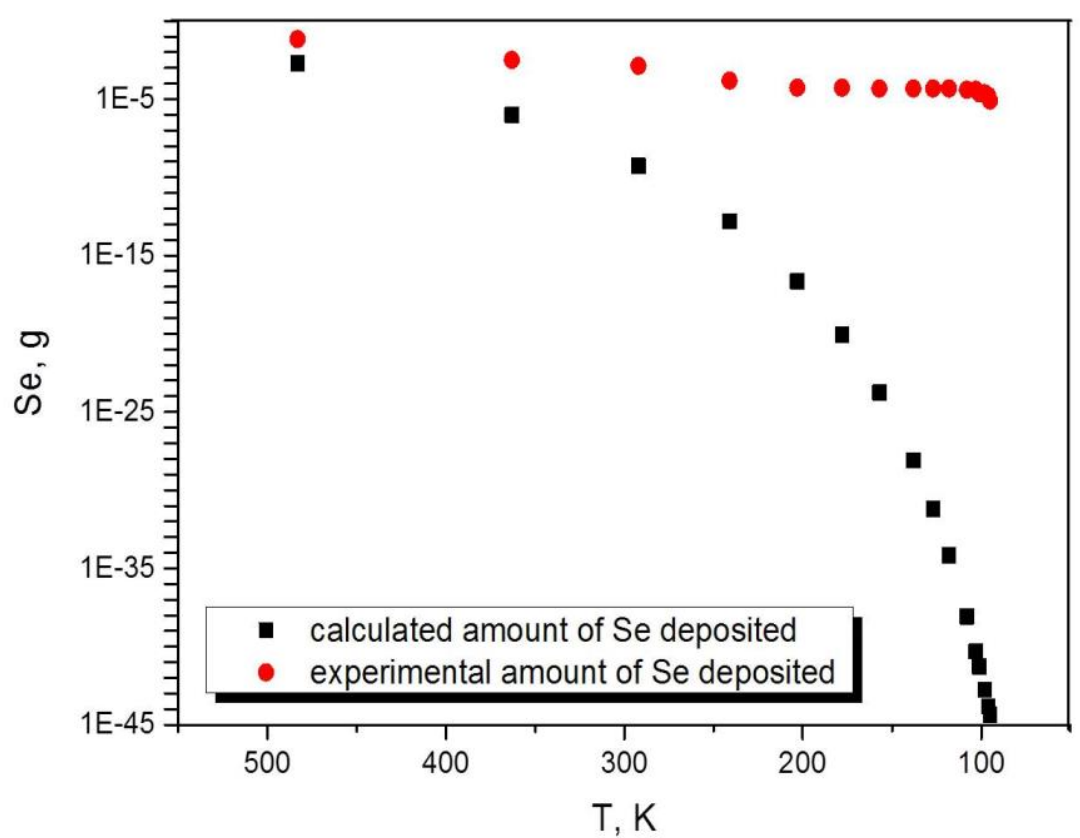

Figure 6. Comparison between the Se deposition pattern a) in this work (red dots); b) calculated according to Antoine's equation for liquid trigonal selenium (black squares). 


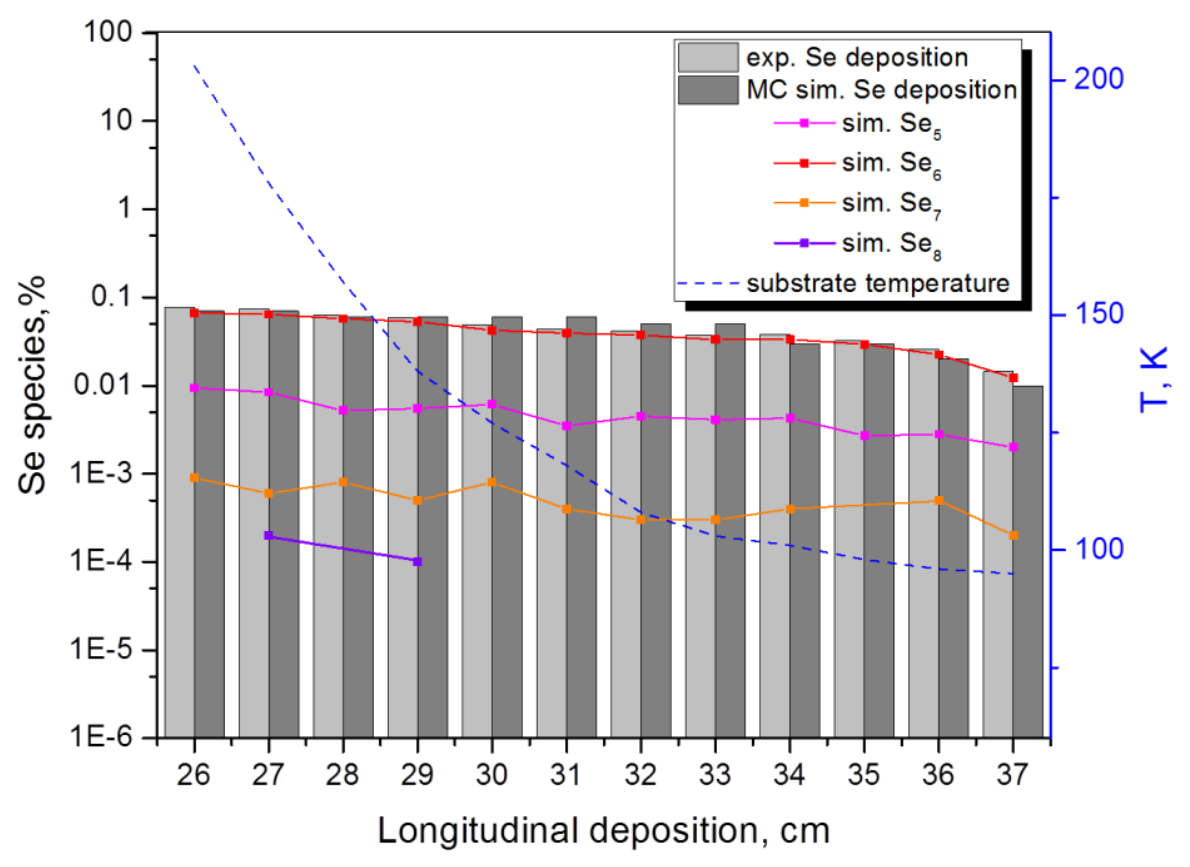

Figure 7. The experimental deposition at $\mathrm{T} \leq 203 \mathrm{~K}$ (i.e. after $\mathrm{cm} 26$ in Fig. 2) of the 
red amorphous Se phase (red line, dots), compared to the Monte-Carlo simulation (black line,

dots) result. The simulated disentangled composition of the deposited phase is indicated (colored lines, squares). 


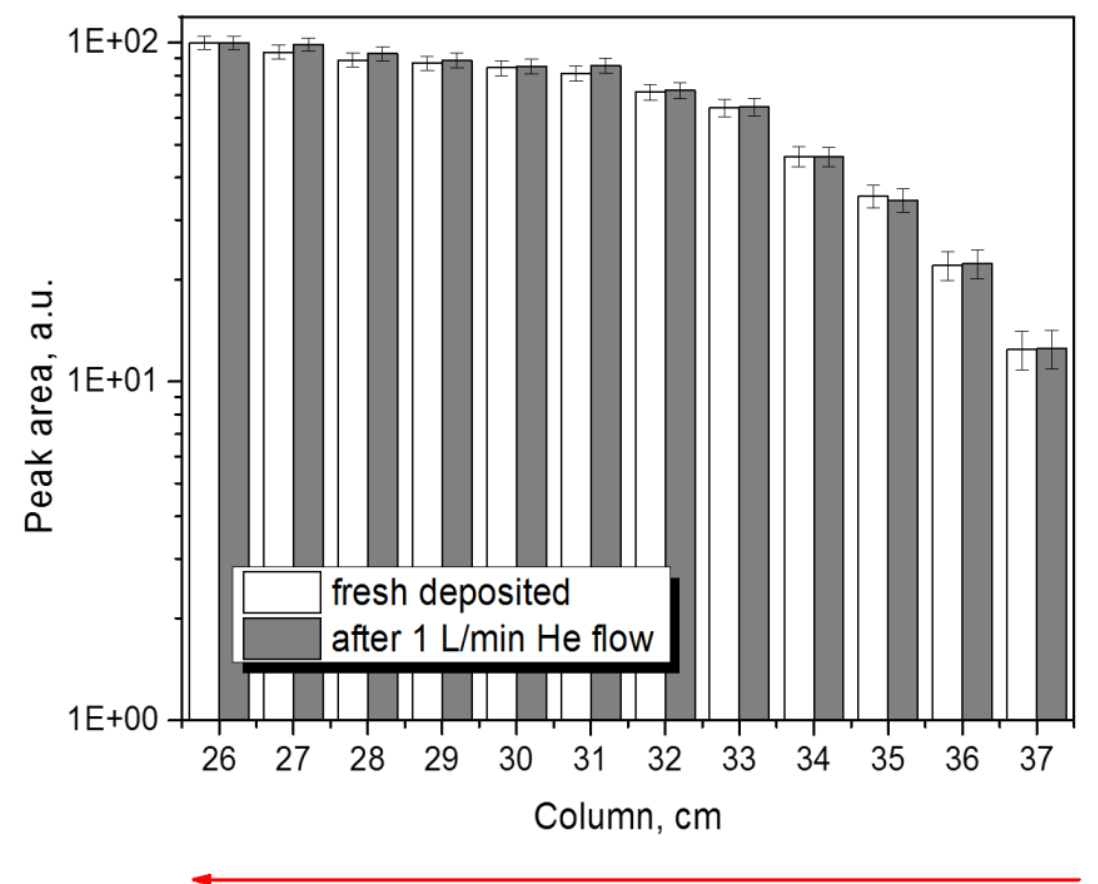

He gas flow direction 
Figure 8 . The area of the ${ }^{75}$ Se peak $(E=136 \mathrm{keV})$ for each centimeter of the column corresponding to the deposited amorphous selenium is given (white bars). After a gas flow exposure of $1 \mathrm{~L} / \mathrm{min}$ during $60 \mathrm{~h}$, the same ${ }^{75} \mathrm{Se} \gamma$-line was measured for each column centimeter (grey bars). Statistical error limits are given at the $68 \%$ confidence interval 